\title{
NATURE OF THE SOFT ULX IN NGC 247: SUPER-EDDINGTON OUTFLOW AND TRANSITION BETWEEN THE SUPERSOFT AND SOFT ULTRALUMINOUS REGIMES
}

\author{
Hua Feng ${ }^{1}$, Lian TaO ${ }^{2}$, Philip KaAret ${ }^{3}$, and Fabien Grisé ${ }^{4}$ \\ ${ }_{1}^{1}$ Department of Engineering Physics and Center for Astrophysics, Tsinghua University, Beijing 100084, China \\ 2 Cahill Center for Astronomy and Astrophysics, California Institute of Technology, Pasadena, CA 91125, USA \\ ${ }^{3}$ Department of Physics and Astronomy, University of Iowa, Iowa City, IA 52242, USA
${ }^{4}$ Observatoire astronomique de Strasbourg, Université Strasbourg, CNRS, UMR 7550, 11 rue de l'Université, F-67000 Strasbourg, France \\ Received 2016 February 24; revised 2016 August 19; accepted 2016 August 24; published 2016 November 3
}

\begin{abstract}
We report on XMM-Newton/Chandra/Swift/Hubble Space Telescope observations of the ultraluminous X-ray source (ULX) in NGC 247, which is found to make transitions between the supersoft ultraluminous (SSUL) regime with a spectrum dominated by a cool $(\sim 0.1 \mathrm{keV})$ blackbody component and the soft ultraluminous (SUL) regime with comparable luminosities shared by the blackbody and power-law components. Multi-epoch observations revealed an anti-correlation between the blackbody radius and temperature, $R_{\mathrm{bb}} \propto T_{\mathrm{bb}}^{-2.8 \pm 0.3}$, ruling out a standard accretion disk as the origin of the soft X-ray emission. The soft X-ray emission is much more variable on both short and long timescales in the SSUL regime than in the SUL regime. We suggest that the SSUL regime may be an extension of the ultraluminous state toward the high accretion end, being an extreme case of the SUL regime, with the blackbody emission arising from the photosphere of thick outflows and the hard X-rays being emission leaked from the embedded accretion disk via the central low-density funnel or advected through the wind. However, the scenario that the supersoft ULXs are standard ULXs viewed nearly edge-on cannot be ruled out. Flux dips on a timescale of $200 \mathrm{~s}$ were observed. The dips cannot be explained by an increase of absorption, but could be due to the change of accretion rate or related to thermal fluctuations in the wind or disk. The optical emission of NGC 247 ULX exhibits a blackbody spectrum at a temperature of $19,000 \mathrm{~K}$ with a radius of $20 R_{\odot}$, likely arising from an OB supergiant companion star.
\end{abstract}

Key words: accretion, accretion disks - black hole physics - galaxies: individual (NGC 247) - X-rays: binaries

\section{INTRODUCTION}

Ultraluminous X-ray sources (ULXs) are non-nuclear accreting X-ray sources that have apparent luminosity that exceeds the Eddington limit of Galactic stellar mass black holes $\left(3 \times 10^{39} \mathrm{erg} \mathrm{s}^{-1}\right.$ for a $20 M_{\odot}$ black hole). Since they were discovered in the 1970s (Fabbiano 1989), their physical nature is still unclear (for a review, see Feng \& Soria 2011). Deep X-ray studies in recent years favor the interpretation that most ULXs are likely due to supercritical accretion onto stellar mass black holes (e.g., Gladstone et al. 2009; Middleton et al. 2012; Sutton et al. 2013; Middleton et al. 2015a), with dynamical evidence in a couple cases (Liu et al. 2013; Motch et al. 2014). Theoretical models and numerical simulations (King \& Pounds 2003; Poutanen et al. 2007; Dotan \& Shaviv 2011; Ohsuga \& Mineshige 2011; Kawashima et al. 2012; Jiang et al. 2014; Shen et al. 2015) suggest that massive outflows are inevitable for supercritical accretion. The outflows may be optically thick and play an important role in shaping the emergent X-ray energy spectrum and timing behavior. It is generally agreed that the disk will be thick and inefficient in radiation, e.g., the slim disk model (Abramowicz et al. 1988). However, recent 3D radiation magnetohydrodynamic simulations of supercritical accretion by Jiang et al. (2014) show that vertical advection induced by turbulence generated by the magnetorotational instability can produce radiative efficiency as high as standard thin-disk accretion and nearly isotropic emission. However, the ULX population is heterogeneous. Extreme ULXs, like ESO 243-49 HLX-1 and M82 X-1 are good candidates for intermediate-mass black holes (Farrell et al. 2009; Feng \& Kaaret 2010; Servillat et al. 2011; Pasham et al. 2014). While a transient ULX in M82 with a peak luminosity over $10^{40} \mathrm{erg} \mathrm{s}^{-1}$ (Feng \& Kaaret 2007a; Brightman et al. 2016) is powered by accretion onto a neutron star (Bachetti et al. 2014). The nature of ULXs is still a question under debate and more observations are needed.

Unlike typical ULXs, which exhibit substantial emission above $2 \mathrm{keV}$, there exists a sub-class for which the X-ray spectrum is dominated by emission from a blackbody-like $\left(k T_{\mathrm{bb}} \approx 0.05-0.2 \mathrm{keV}\right.$ ) component below $2 \mathrm{keV}$ (Di Stefano \& Kong 2003, 2004). Well studied examples include those in M51 (Di Stefano \& Kong 2003; Terashima \& Wilson 2004; Dewangan et al. 2005; Terashima et al. 2006), M81 (Swartz et al. 2002, 2003; Liu 2008; Liu \& Di Stefano 2008; Liu et al. 2015), M101 (Pence et al. 2001; Mukai et al. 2003; Jenkins et al. 2004; Kong et al. 2004; Kong \& Di Stefano 2005; Mukai et al. 2005; Liu 2009; Liu et al. 2013; Shen et al. 2015; Soria \& Kong 2016), NGC 247 (Winter et al. 2006; Jin et al. 2011; Tao et al. 2012a), NGC 300 (Read \& Pietsch 2001; Kong \& Di Stefano 2003), the Antennae (NGC 4038/4039; Fabbiano et al. 2003), and NGC 4631 (Carpano et al. 2007; Soria \& Ghosh 2009). Multi-epoch observations of the canonical soft ULX in M101 revealed dramatic variability in the observed X-ray flux by two to three orders of magnitude (Soria \& Kong 2016). Such strong variability is rare for broadband ULXs.

Urquhart \& Soria (2016) performed a comprehensive X-ray study of the brightest soft ULXs. They found a correlation between the blackbody temperature and emission radius, $R_{\mathrm{bb}} \propto T_{\mathrm{bb}}^{-2.2 \pm 0.5}$, for their sample. Soria \& Kong (2016) identified a similar relation, roughly $R_{\mathrm{bb}} \propto T_{\mathrm{bb}}^{-2}$, for the soft ULX in M101 from multi-epoch observations. This seems to be a universal relation for soft ULXs. It has been proposed that the 
Table 1

Observations Used in the Paper

\begin{tabular}{|c|c|c|c|c|}
\hline Telescope & ID & Date & Exposure & Detector/Filter \\
\hline XMM-Newton & 0601010101 & 2009 Dec 27-28 & $30 \mathrm{ks}$ & MOS1/MOS2/PN \\
\hline XMM-Newton & 0728190101 & 2014 Jul 01 & $30 \mathrm{ks}$ & MOS1/MOS2/PN \\
\hline Chandra & 12437 & 2011 Feb 01 & $5.0 \mathrm{ks}$ & ACIS-S \\
\hline Chandra & 17547 & 2014 Nov 12 & $5.0 \mathrm{ks}$ & ACIS-S \\
\hline Swift & 00033469 & 2014 Oct-2015 Nov & $28 \times 2 \mathrm{ks}$ & XRT \\
\hline$H S T$ & ic8ea1010 & 2014 Jun 30 & $468 \mathrm{~s}$ & WFC3/UVIS/F225W \\
\hline$H S T$ & ic8ea1020 & 2014 Jun 30 & $466 \mathrm{~s}$ & WFC3/UVIS/F336W \\
\hline$H S T$ & ic8ea1030 & 2014 Jun 30 & $96 \mathrm{~s}$ & WFC3/UVIS/F438W \\
\hline$H S T$ & ic8ea1040 & 2014 Jun 30 & $96 \mathrm{~s}$ & WFC3/UVIS/F606W \\
\hline$H S T$ & ic8ea1050 & 2014 Jun 30 & $200 \mathrm{~s}$ & WFC3/UVIS/F814W \\
\hline$H S T$ & ic8ea1060 & 2014 Jun 30 & $257 \mathrm{~s}$ & WFC3/IR/F105W \\
\hline$H S T$ & ic8ea1070 & 2014 Jun 30 & $303 \mathrm{~s}$ & WFC3/IR/F160W \\
\hline$H S T$ & jc8e01020 & 2014 Jun 30 & $2348 \mathrm{~s}$ & $\mathrm{ACS} / \mathrm{SBC} / \mathrm{PR} 130 \mathrm{~L}$ \\
\hline
\end{tabular}

soft spectrum of soft ULXs could be emission from the photosphere of optically thick outflows, which obscure or soften the hard emission from the inner disk (Feng \& Soria 2011; Shen et al. 2015; King \& Muldrew 2016; Soria \& Kong 2016). King \& Muldrew (2016) suggest that the soft ULXs and broadband ULXs are the same type of objects viewed at different angles. Urquhart \& Soria (2016) suggest that the soft ULXs could be a special state of the normal ULXs; they may transition to be broadband ULXs when the photosphere becomes smaller and hotter $\left(k T_{\mathrm{bb}} \sim 150 \mathrm{eV}\right)$. These scenarios have yet to be tested because no source has been observed to date to transition between the two states or regimes.

In this paper, we report multiwavelength observations of one of the brightest soft ULXs in NGC 247 (R.A. $=00^{\mathrm{h}} 47^{\mathrm{m}} 03^{\mathrm{s}} .88$, decl. $=-20^{\circ} 47^{\prime} 44^{\prime \prime} 3$, J2000.0). It was first tagged as a ULX from a short and badly contaminated XMM-Newton observation (Winter et al. 2006). Later, its soft nature was secured via a second XMM-Newton observation (Jin et al. 2011). The optical counterpart to the X-ray source was identified using aligned Chandra and Hubble Space Telescope (HST) images (Tao et al. 2012a). Here, we attempt to unveil the physical origin of the optical emission using multi-band HST observations and the nature of the X-ray emission using multi-epoch observations with XMM-Newton, Chandra, and Swift. We adopt a distance of $3.4 \mathrm{Mpc}$ estimated using IR Cepheids to the galaxy NGC 247 (Gieren et al. 2009).

We use the term soft instead of supersoft to refer to these sources, in particular, for NGC 247 ULX, because they are always soft but not always supersoft. They vary in temperature and spectral shape, and some of them in some occasions may display substantial emission between 1 and $2 \mathrm{keV}$ or even above $2 \mathrm{keV}$. In their hardest state, they may not be classified as supersoft sources based on the criteria defined by Di Stefano \& Kong (2003) due to the presence of a relatively strong power-law component.

\section{OBSERVATIONS AND DATA REDUCTION}

The observations used in this paper are listed in Table 1. NGC 247 was observed with XMM-Newton on 2001 July 08 (ObsID 0110990301), 2009 December 07 (ObsID 0601010101), and 2014 July 01 (ObsID 0728190101). The first observation, from which the source was found to exhibit a soft spectrum (Winter et al. 2006; Jin et al. 2011), was short and strongly contaminated by background flares and is not considered for spectral analysis here. The second observation has been previously described by Jin et al. (2011), who confirmed that the source spectrum consists of a dominant soft, thermal component and a weak power-law component, reminiscent of the thermal state spectrum of Galactic black hole binaries. The third observation was obtained as of our multiwavelength campaign with HST and is described in detail below. In addition to the sensitive XMM-Newton observations, we also report results from two short Chandra observations (5 ks each) and 28 Swift snapshots ( $2 \mathrm{ks}$ each) of the source.

\subsection{XMM-Newton}

New event files were produced with updated calibration files. Clean exposures were selected from time intervals where the background flux is within $\pm 3 \sigma$ of the mean quiescent level. For the 2009 observation (ObsID 0601010101), the total clean exposure is $22.3 \mathrm{ks}$ for PN, $29.0 \mathrm{ks}$ for MOS1, and $30.2 \mathrm{ks}$ for MOS2. For the 2014 observation (ObsID 0728190101), the exposure is $27.2 \mathrm{ks}$ for PN, $31.0 \mathrm{ks}$ for MOS1, and $32.4 \mathrm{ks}$ for MOS2.

Figure 1 shows PN light curves for the longest (26.1 ks) continuous and low background time interval from the 2014 observation. Events were extracted from a $32^{\prime \prime}$-radius circular region surrounding the source and selected to have FLAG $=$ \#XMMEA_EP and PATTERN $\leqslant 4$. The background contribution was estimated from several nearby regions and subtracted. Light curves are displayed in the full band of $0.2-10 \mathrm{keV}$, a soft band of $0.2-0.75 \mathrm{keV}$, a medium band of $0.75-1.5 \mathrm{keV}$, and a hard band of $1.5-10 \mathrm{keV}$. The bottom panel of Figure 1 shows hardness ratios, defined as $\mathrm{HR} 1=($ medium - soft $) /($ medium + soft $)$ and HR2 $=($ hard - medium - soft $) /($ hard + medium + soft $)$. HR1 is sensitive to the temperature of the soft thermal component and HR2 is sensitive to the flux ratio of the hard power-law component versus the soft thermal component. As one can see, the source displayed a dramatic change in flux (dips) and the medium-to-soft hardness ratio. Time intervals for the low- and high-flux states are marked in Figure 1; their energy spectra were extracted and analyzed separately.

The same aperture was used to extract the source spectra and a few nearby circular regions on the same CCD chip at similar readout distances, but off the readout direction were used to 


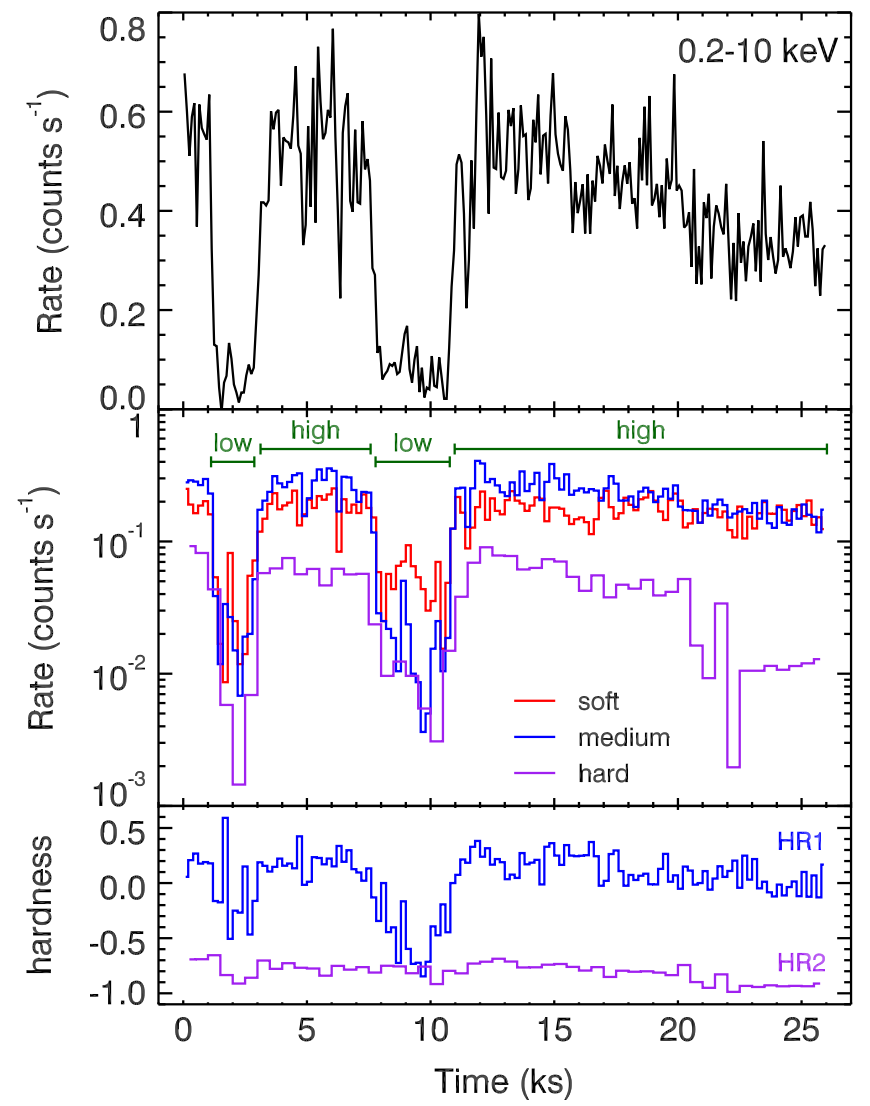

Figure 1. XMM-Newton PN light curves of NGC 247 ULX from the 2014 observation in the longest continuous and clean time interval with an exposure of $26.1 \mathrm{ks}$. Top: light curve in the full band $(0.2-10 \mathrm{keV})$. Middle: light curves in the soft band $(0.2-0.75 \mathrm{keV})$, medium band $(0.75-1.5 \mathrm{keV})$, and hard band $(1.5-10 \mathrm{keV})$. Bottom: hardness ratios defined as $\mathrm{HR} 1=($ medium $-\mathrm{soft}) /$ $($ medium + soft $)$ and HR2 $=($ hard - medium - soft $) /($ hard + medium + soft). The light curve has a time step of $100 \mathrm{~s}$ for the full band, $200 \mathrm{~s}$ for the soft and medium bands, and $500 \mathrm{~s}$ for the hard band.

extract the background spectra, both from events with FLAG $=0$ and PATTERN $\leqslant 12$ for MOS or $\leqslant 4$ for PN. Each spectrum was grouped in the energy band from $0.2 \mathrm{keV}$ to roughly $10 \mathrm{keV}$ to have 4 bins per (FWHM) spectral resolution element or at least 25 counts.

\subsection{Chandra}

New level 2 event files were created using the repro script in CIAO. An elliptical source region found by the wavdetect task was adopted for spectrum extraction, which was 1".62 in semimajor radius and 1 ". 15 in semiminor radius for the 2011 observation (ObsID 12437), and 1".46 and 1".32, respectively, for the 2014 observation (ObsID 17547). A nearby large, source-free region was used to extract a background spectrum. For ObsID 12437, there are 160 counts detected in the energy band of $0.3-1 \mathrm{keV}, 7$ counts in $1-2 \mathrm{keV}$, and only 1 count in $2-8 \mathrm{keV}$. For ObsID 17547, there are 103 counts in $0.3-1 \mathrm{keV}$, 70 counts in $1-2 \mathrm{keV}$, and 4 counts in $2-8 \mathrm{keV}$. Thus, in order to perform $\chi^{2}$ fitting, we grouped the energy spectrum by at least 15 counts per bin from 0.3 to $1 \mathrm{keV}$ for ObsID 12437, and in the same manner from 0.3 to $2 \mathrm{keV}$ for ObsID 17547. This results in eight energy bins for ObsID 12437 and 9 for ObsID 17547.

\subsection{Swift}

The NGC 247 galaxy was monitored with the Swift X-ray telescope (XRT) from 2014 October 13 to 2015 November 19 under the program 00033469. The cadence was a week in the beginning (once interrupted due to Sun avoidance) and changed to a month for the last six observations. The requested exposure for each observation was $2 \mathrm{ks}$, but it varied from 0.9 to $2.5 \mathrm{ks}$. The detected photons in the source aperture ranges from 8 to 51 for a single observation, and the background contribution is typically $3 \%$, but $11 \%$ in the worst case. The source displayed a correlation between count rate and hardness ratio in individual XRT observations. Thus, in order to perform $\chi^{2}$ fitting, we combined events from observations with similar background corrected count rates. We divided the 28 individual observations into five groups, each one with a total number of 120-130 source counts. We extracted the source spectrum from an aperture of 20 pixels in radius from the combined event files. The background spectrum was extracted from a nearby sourcefree region, verified using the deep XMM-Newton image. Each spectrum was grouped to have at least 15 counts per bin from 0.3 to $2 \mathrm{keV}$.

\subsection{HST}

The HST observations (proposal ID 13425) started about $18 \mathrm{hr}$ before the beginning of the XMM-Newton observation and were finished in two orbits $(\sim 2.4 \mathrm{hr})$. Images were taken using the Wide Field Camera 3 (WFC3) with both the UVIS and IR Channels. A number of wide-band filters (see Table 1) were used to cover a wavelength range from 1900 to $18000 \AA$. A $512 \times 512$ subarray was adopted for both UVIS and IR to reduce the readout time. Images around the ULX region for different filters are shown in Figure 2. Point spread function (PSF) photometry was performed on the calibrated, flat-fielded images using the DOLPHOT 2.0 package (Dolphin 2000). We first ran wfc3mask to mask bad pixels, and then split UVIS images using splitgroups. After estimating the sky value with calcsky, we ran dolphot to get the aperture and charge transfer efficiency corrected Vega magnitudes. We compared with aperture photometry and found consistent results in the UVIS bands. For IR images, the ULX region is crowded with faint sources and the difference in PSF and aperture photometry is as large as $0.7 \mathrm{mag}$ in the F160W band. We thus adopted results from the PSF photometry, which should produce more reliable results for sources in crowded regions, but note that the true errors for IR flux taking into account systematics in photometry may be larger. The SYNPHOT package was used for flux conversion. The observed magnitudes in different bands are listed in Table 2.

The low-resolution grism PR130L in the Solar Blind Channel (SBC) of the Advanced Camera for Surveys (ACS) was used to produce a slitless spectrum in the UV band. The dispersed image of the PR130L prism (see Figure 3) consists of four sub-exposures. The last sub-exposure (jc8e01e3q) had a low signal-to-noise ratio $(\mathrm{S} / \mathrm{N})$ due to an increase of detector temperature and was not used. Using version 2.4.4 of the aXe software package in PyRAF/STSDAS, spectra were extracted with an aperture width of 12 pixels. The direct image, taken through the F165LP filter, was used to provide the wavelength zero point on the prism image. The spectrum covers the wavelength range from 1300 to $1900 \AA$. Due to the faintness of the object, no significant emission or absorption line features 


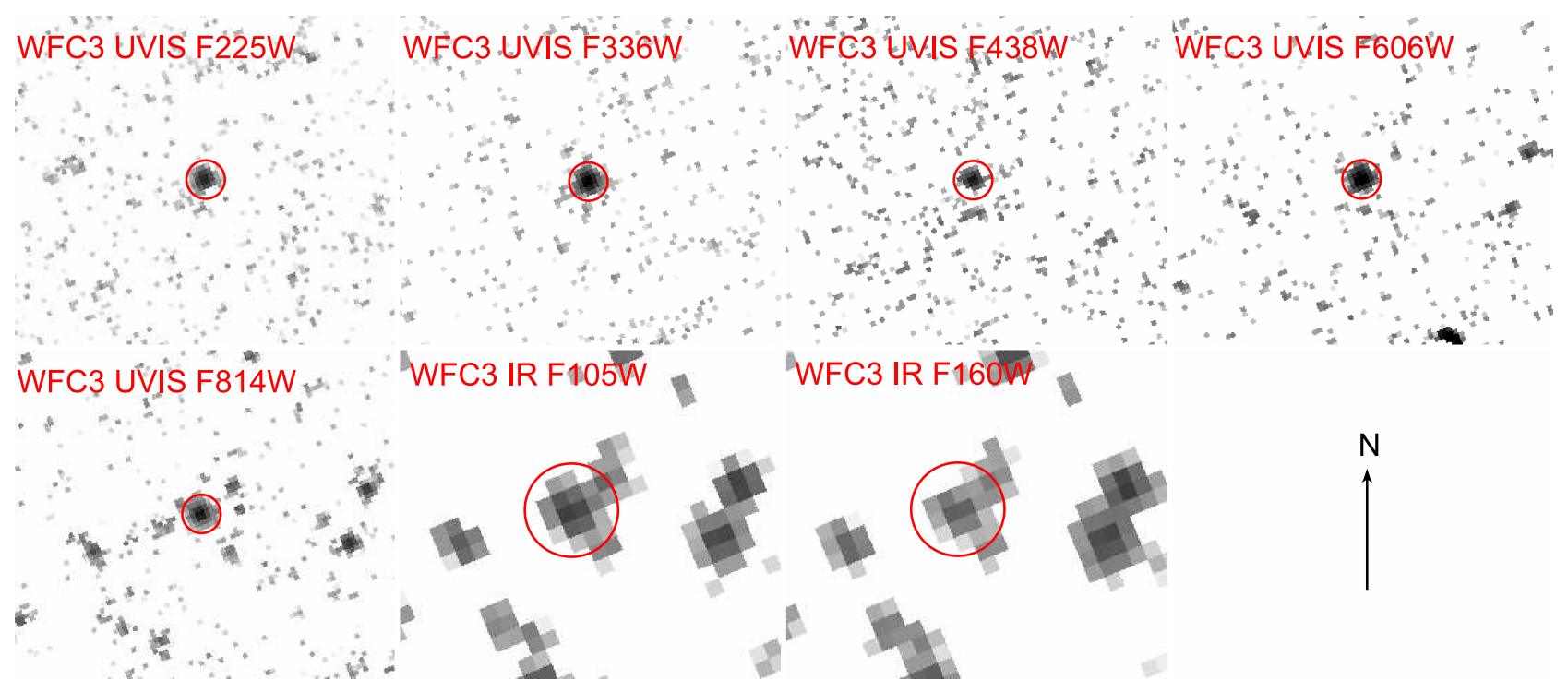

Figure 2. HST WFC3 images around the ULX region. The circles indicate the optical counterpart of the ULX. The arrow points north and has a length of $1^{\prime \prime}$.

Table 2

Observed Magnitudes of the ULX with HST WFC3

\begin{tabular}{lccc}
\hline \hline Channel & Filter & Pivot Wavelength $(\AA)$ & Vega mag \\
\hline UVIS & F225W & 2359 & $20.77 \pm 0.03$ \\
UVIS & F336W & 3355 & $21.03 \pm 0.02$ \\
UVIS & F438W & 4325 & $22.47 \pm 0.05$ \\
UVIS & F606W & 5887 & $22.22 \pm 0.03$ \\
UVIS & F814W & 8024 & $22.07 \pm 0.03$ \\
IR & F105W & 10552 & $21.67 \pm 0.02$ \\
IR & F160W & 15369 & $21.60 \pm 0.03$ \\
\hline
\end{tabular}

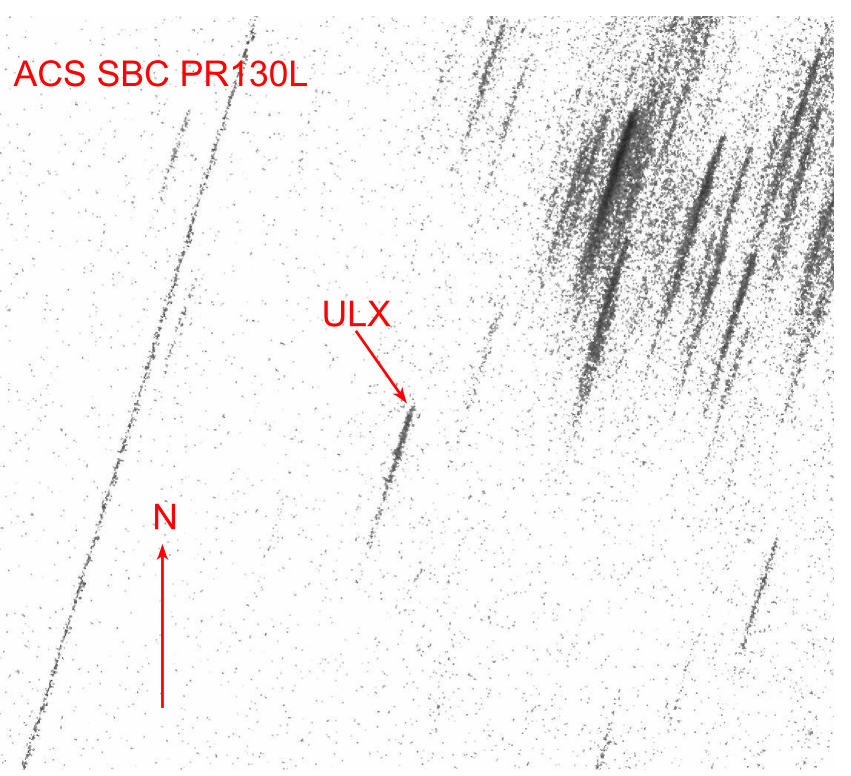

Figure 3. Dispersed image from the grism ACS SBC PR130L around the ULX. The arrow points north and has a length of $3^{\prime \prime}$.

can be detected. The three spectra from the sub-exposures were stacked and binned to have an $\mathrm{S} / \mathrm{N}$ of at least 15 per spectral bin (except for the last bin, which has an $\mathrm{S} / \mathrm{N}$ of 9.4).

\section{ANALYSIS AND RESULTS}

\subsection{Spectral Modeling to the X-Ray Data}

The XMM-Newton PN and MOS spectra from the 2014 observation were fitted jointly using XSPEC. A constant scaling factor on the MOS flux with respect to the PN flux is included for the high-state spectra as a free parameter to account for possible difference in their absolute flux, and is found to be close to unity within $2 \%$; for the low-state spectra, the constant is fixed at unity due to low statistics. The TBabs model (Wilms et al. 2000) is used to model the interstellar absorption, with a Milky Way component fixed at $N_{\mathrm{H}}=2.06 \times 10^{20} \mathrm{~cm}^{-2}$ (Kalberla et al. 2005) and a free, extragalactic component.

Following Jin et al. (2011), two models were used to fit the soft thermal emission, a multicolor disk (MCD; Shakura \& Sunyaev 1973) and a mono-temperature blackbody. A powerlaw component is added to account for the hard tail in the spectrum. A combination of an MCD and a power law is standard for modeling the X-ray spectrum of accreting black hole binaries, while the choice of a mono-temperature blackbody is appropriate if the soft thermal emission arises from the photosphere of thick outflows.

For the high-state spectra from the 2014 observation (ObsID 0728190101), the fitting is significantly improved if an optically thin thermal plasma model (APEC) is added to the two-component model. The F-test suggests that the inclusion of the APEC model has a chance probability of $\sim 3 \times 10^{-6}$. Inspection of the residuals, especially for the PN data, shows an excess around $0.6 \mathrm{keV}$. To account for that feature, we added a zero-width Gaussian; the F-test chance probability is $2 \times 10^{-5}$.

For the low-state spectra in the 2014 observation, the MCD/ blackbody plus power-law model gives an adequate fit and presence of additional components cannot be justified due to the limited statistics. The low statistics also prevent the fitting from breaking the degeneracy between the absorption column density and the temperature of the thermal component. Keeping both parameters free, the fitting converges to a combination of strong absorption $\left(\sim 10^{22} \mathrm{~cm}^{-2}\right)$ and a cool, large-area soft thermal component with an unphysically large bolometric luminosity $\left(\sim 10^{42} \mathrm{erg} \mathrm{s}^{-1}\right)$. Thus, we fixed the extragalactic 
absorption column density at $4 \times 10^{21} \mathrm{~cm}^{-2}$, the typical value derived from the high-quality data. If we adopt the best-fit model derived from the high-state spectra and fix all parameters except the extragalactic absorption column density, no successful fit can be obtained, with a reduced $\chi^{2} \sim 13$. Thus, we conclude that the dipping is not a result of increased absorption alone.

The spectral analysis for the 2009 observation (ObsID 0601010101) has been reported in detail in Jin et al. (2011). The best-fit spectral model consists of two major components, a blackbody or MCD component dominant below $2 \mathrm{keV}$ and a power-law component dominant above $2 \mathrm{keV}$. In addition, the inclusion of an absorption edge near $1 \mathrm{keV}$ improved the fit, with a chance probability of $1 \times 10^{-4}$ estimated via simulation. Thus, for consistency, we extracted the energy spectra in the same manner as above and fitted them using the best-fit model found by Jin et al. (2011), i.e., edge $*($ MCD/ blackbody + power-law) subject to both Galactic and extragalactic absorption (TBabs). Here we use both PN and MOS data, while Jin et al. (2011) used PN data only. Our results are consistent within 1 or $2 \sigma$ errors. The X-ray spectra with model components are displayed in Figure 4 and all the model parameters are tabulated in Table 3. The observed flux and intrinsic luminosity was calculated using the cflux command in XSPEC. The factor for absorption-correction is $\sim 10$ for the 2014 high-state spectra, $\sim 15$ for the low-state spectra, and $\sim 20$ for the 2009 spectra.

For the 2014 high-state spectra, $45 \%$ of the intrinsic luminosity in the energy range of $0.3-10 \mathrm{keV}$ arises from the blackbody component, $45 \%$ from the power-law component, $3 \%$ from the APEC component, and 6\% from the Gaussian line. For the 2014 low state and 2009 spectra, the blackbody component is dominant and has $\sim 95 \%$ of the luminosity in the same band.

For the Chandra spectra, a single blackbody or MCD spectrum subject to interstellar absorption (TBabs) is adequate to fit the spectrum in the $0.3-1 \mathrm{keV}$ band (for ObsID 12437) or the $0.3-2 \mathrm{keV}$ band (for ObsID 17547). The best-fit results are listed in Table 4 . The extragalactic absorption column density is fixed at the typical value, $4 \times 10^{21} \mathrm{~cm}^{-2}$, found from the $X M M-N e w t o n$ data. If it is allowed to vary, the best-fit value is around $(2-5) \times 10^{21} \mathrm{~cm}^{-2}$ but the error bounds of this and other parameters cannot be well constrained, due to the limitation of data quality. Each combined Swift/XRT spectrum is fitted in the same way as for the Chandra spectra. The results are presented in Table 5.

\subsection{Long-term X-Ray Variability and Spectral Evolution}

The best-fit blackbody temperature and radius for each observation or observation group is shown in Figure 5. For $X M M-N e w t o n$ data, results from the most sophisticated models (the best model) are adopted and the absorption column density is a free parameter, while for Chandra and Swift data, due to the low quality of data, the column density is fixed. The small errors on Chandra and Swift results are due to the fact that the absorption component was fixed and its large degeneracy with the soft blackbody component was vanished. A linear correlation is seen between the blackbody temperature and radius on a logarithmic scale. A linear regression using the bivariate correlated errors and intrinsic scatter (BCES) algorithm (Akritas \& Bershady 1996), which considers errors on both $x$ and $y$ in the type of $y \mid x$ (to predict $y$ using $x$ ), suggests a
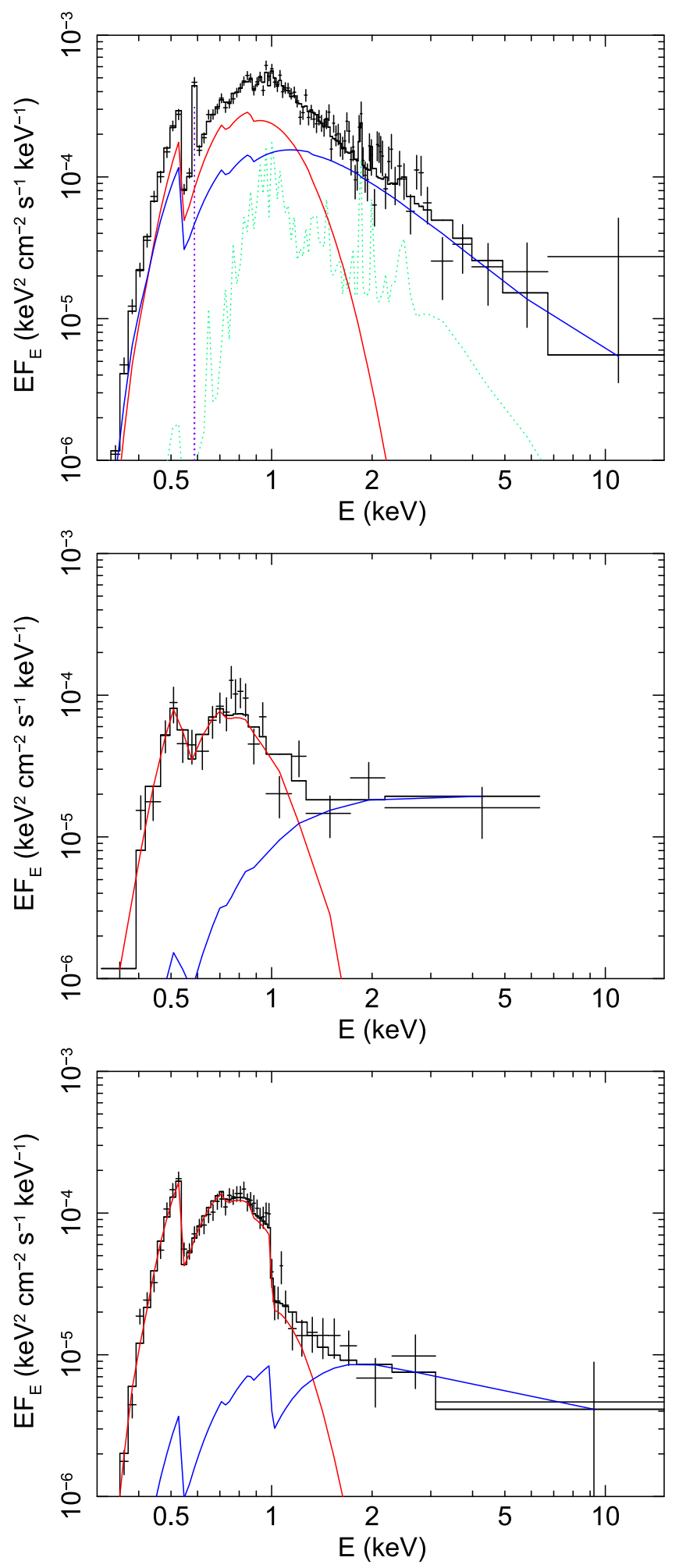

Figure 4. Unfolded X-ray spectra and best-fit models for NGC 247. Top: the 2014 observation in the high state; the model consists of a blackbody component (red), a power-law component (blue), a thermal plasma (APEC) component (green), and an emission line (purple). Middle: the 2014 observation in the low state, fit with a blackbody plus power-law model with fixed absorption column density. Bottom: the 2009 spectrum decomposed into a blackbody plus a powerlaw component, both subject to an absorption edge near $1 \mathrm{keV}$.

relation $R_{\mathrm{bb}} \propto T_{\mathrm{bb}}^{-2.8 \pm 0.3}$. We also plot model predicted relations assuming super-Eddington accretion with massive outflows (Soria \& Kong 2016) to compare with the data. 
Table 3

Spectral Modeling to the XMM-Newton Spectra

\begin{tabular}{|c|c|c|c|c|c|c|c|c|c|c|c|c|}
\hline Model & $\begin{array}{c}N_{\mathrm{H}, \mathrm{ext}}{ }^{\mathrm{a}} \\
\left(10^{21} \mathrm{~cm}^{-2}\right)\end{array}$ & $\begin{array}{c}k T_{\mathrm{th}} \\
(\mathrm{keV})\end{array}$ & $\begin{array}{c}R_{\mathrm{th}}{ }^{\mathrm{b}} \\
\left(10^{4} \mathrm{~km}\right)\end{array}$ & $\Gamma$ & $N_{\mathrm{PL}}^{\mathrm{c}}$ & $\begin{array}{c}k T_{\mathrm{APEC}} \\
(\mathrm{keV})\end{array}$ & $\begin{array}{c}E_{\text {line }} \\
(\mathrm{keV})\end{array}$ & $N_{\text {line }}^{\mathrm{d}}$ & $\begin{array}{c}f_{0.3-10} \mathrm{keV} \\
\left(10^{-13} \mathrm{cgs}\right)\end{array}$ & $\begin{array}{l}L_{0.3}-10 \mathrm{keV} \\
\left(10^{40} \mathrm{cgs}\right)\end{array}$ & $\begin{array}{c}L_{\text {tho }} \text { bol } \\
\left(10^{40} \mathrm{cgs}\right)\end{array}$ & $\chi^{2} /$ dof \\
\hline \multicolumn{13}{|c|}{ ObsID 0728190101: High State } \\
\hline A & $4.5_{-0.5}^{+0.5}$ & $0.145_{-0.012}^{+0.013}$ & $1.5_{-0.4}^{+0.7}$ & $3.9_{-0.3}^{+0.3}$ & $4.9_{-1.3}^{+1.6}$ & $\ldots$ & $\ldots$ & $\cdots$ & $7.90_{-0.17}^{+0.13}$ & $1.7_{-0.5}^{+0.7}$ & 1.2 & $320.02 / 270$ \\
\hline B & $3.7_{-0.6}^{+0.6}$ & $0.156_{-0.017}^{+0.019}$ & $0.9_{-0.4}^{+0.6}$ & $3.7_{-0.4}^{+0.4}$ & $3.2_{-1.1}^{+1.4}$ & $1.01_{-0.08}^{+0.12}$ & $\cdots$ & $\cdots$ & $7.94_{-0.18}^{+0.07}$ & $1.0_{-0.3}^{+0.6}$ & 0.69 & $290.40 / 268$ \\
\hline $\mathrm{C}$ & $4.2_{-0.7}^{+0.8}$ & $0.148_{-0.017}^{+0.021}$ & $1.2_{-0.5}^{+0.5}$ & $3.9_{-0.4}^{+0.4}$ & $3.7_{-1.3}^{+1.7}$ & $0.98_{-0.05}^{+0.12}$ & $0.585_{-0.011}^{+0.010}$ & $6_{-3}^{+6}$ & $7.95_{-0.21}^{+0.11}$ & $1.4_{-0.6}^{+1.1}$ & 0.94 & $267.48 / 266$ \\
\hline$A^{\prime}$ & $4.0_{-0.5}^{+0.5}$ & $0.128_{-0.009}^{+0.010}$ & $1.5_{-0.4}^{+0.6}$ & $3.9_{-0.3}^{+0.3}$ & $4.9_{-1.2}^{+1.5}$ & $\cdots$ & $\cdots$ & $\cdots$ & $7.91_{-0.17}^{+0.13}$ & $1.1_{-0.3}^{+0.5}$ & 0.77 & $313.55 / 270$ \\
\hline $\mathrm{B}^{\prime}$ & $3.5_{-0.6}^{+0.6}$ & $0.131_{-0.012}^{+0.013}$ & $1.2_{-0.4}^{+0.6}$ & $3.8_{-0.4}^{+0.4}$ & $3.7_{-1.1}^{+1.4}$ & $1.05_{-0.09}^{+0.13}$ & $\ldots$ & $\ldots$ & $7.93_{-0.15}^{+0.16}$ & $0.8_{-0.3}^{+0.5}$ & 0.56 & $290.40 / 268$ \\
\hline $\mathrm{C}^{\prime}$ & $3.9_{-0.7}^{+0.8}$ & $0.126_{-0.013}^{+0.014}$ & $1.5_{-0.5}^{+1.0}$ & $4.0_{-0.4}^{+0.4}$ & $4.2_{-1.1}^{+1.2}$ & $1.01_{-0.09}^{+0.13}$ & $0.583_{-0.013}^{+0.013}$ & $5_{-3}^{+5}$ & $7.91_{-0.15}^{+0.15}$ & $1.1_{-0.4}^{+0.8}$ & 0.69 & $264.01 / 266$ \\
\hline \multicolumn{13}{|c|}{ ObsID 0728190101: Low State } \\
\hline A & 4.0 fixed & $0.110_{-0.007}^{+0.007}$ & $1.7_{-0.4}^{+0.5}$ & $2.1_{-0.8}^{+0.9}$ & $0.22_{-0.12}^{+0.19}$ & $\cdots$ & $\cdots$ & $\cdots$ & $1.6_{-0.3}^{+0.4}$ & $0.36_{-0.05}^{+0.05}$ & 0.56 & $42.68 / 29$ \\
\hline $\mathrm{A}^{\prime}$ & 4.0 fixed & $0.092_{-0.005}^{+0.005}$ & $2.4_{-0.4}^{+0.6}$ & $2.1_{-0.7}^{+0.9}$ & $0.25_{-0.13}^{+0.19}$ & $\cdots$ & $\cdots$ & $\cdots$ & $1.5_{-0.3}^{+0.4}$ & $0.32_{-0.04}^{+0.05}$ & 0.54 & $39.69 / 29$ \\
\hline \multicolumn{13}{|c|}{ ObsID 0601010101} \\
\hline & & & & & & & $E_{\text {edge }}$ & $\tau_{\text {edge }}$ & & & & \\
\hline \multicolumn{13}{|c|}{$(\mathrm{keV})$} \\
\hline $\mathrm{D}$ & $4.5_{-0.9}^{+1.1}$ & $0.108_{-0.014}^{+0.016}$ & $2.8_{-1.5}^{+3.8}$ & $2.8_{-1.0}^{+1.7}$ & $0.21_{-0.12}^{+0.44}$ & $\cdots$ & $0.994_{-0.018}^{+0.019}$ & $1.3_{-0.4}^{+0.4}$ & $1.68_{-0.11}^{+0.16}$ & $0.8_{-0.4}^{+1.2}$ & 1.4 & $129.01 / 110$ \\
\hline $\mathrm{D}^{\prime}$ & $4.0_{-0.9}^{+1.2}$ & $0.094_{-0.012}^{+0.013}$ & $2.9_{-1.5}^{+3.7}$ & $3.1_{-1.1}^{+2.8}$ & $0.25_{-0.15}^{+1.15}$ & $\cdots$ & $0.996_{-0.020}^{+0.022}$ & $1.2_{-0.4}^{+0.6}$ & $1.65_{-0.12}^{+0.16}$ & $0.5_{-0.3}^{+0.8}$ & 0.88 & $132.48 / 110$ \\
\hline
\end{tabular}

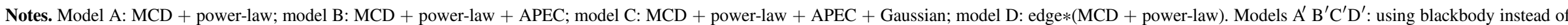

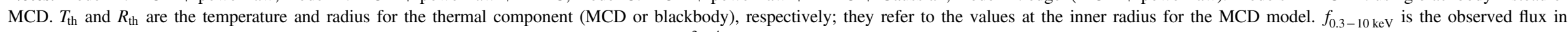
$0.3-10 \mathrm{keV} ; L_{0.3-10 \mathrm{keV}}$ is the absorption-corrected luminosity in $0.3-10 \mathrm{keV} ; L_{\mathrm{th}}$,bol $\equiv 4 \pi \sigma R_{\mathrm{th}}^{2} T_{\mathrm{th}}^{4}$ is the bolometric luminosity for the thermal component. All errors are quoted in the $90 \%$ confidence level.

${ }^{a}$ Extragalactic absorption column density; an additional absorption component fixed at $N_{\mathrm{H}}=2.06 \times 10^{20} \mathrm{~cm}^{-2}$ to account for Galactic absorption is not listed here.

${ }^{\mathrm{b}}$ Assuming a face-on disk.

${ }^{c}$ Normalization for the power-law component in units of $10^{-4}$ photons $\mathrm{keV}^{-1} \mathrm{~cm}^{-2} \mathrm{~s}^{-1}$ at $1 \mathrm{keV}$.

${ }^{\mathrm{d}}$ Normalization for the Gaussian component in units of $10^{-4}$ photons $\mathrm{cm}^{-2} \mathrm{~s}^{-1}$. 
Table 4

Spectral Modeling to the Chandra Spectra

\begin{tabular}{|c|c|c|c|c|c|c|c|c|}
\hline ObsID & Model & $\frac{N_{\mathrm{H}, \mathrm{ext}}}{\left(10^{21} \mathrm{~cm}^{-2}\right)}$ & $\begin{array}{c}k T_{\mathrm{th}} \\
(\mathrm{keV})\end{array}$ & $\begin{array}{c}R_{\mathrm{th}} \\
\left(10^{4} \mathrm{~km}\right)\end{array}$ & $\begin{array}{c}f_{0.3-10} \mathrm{keV} \\
\left(10^{-13} \mathrm{cgs}\right)\end{array}$ & $\begin{array}{l}L_{0.3}-10 \mathrm{keV} \\
\left(10^{40} \mathrm{cgs}\right)\end{array}$ & $\begin{array}{c}L_{\text {th }} \text { bol } \\
\left(10^{40} \mathrm{cgs}\right)\end{array}$ & $\chi^{2} /$ dof \\
\hline 12437 & MCD & 4.0 fixed & $0.105_{-0.009}^{+0.011}$ & $3.1_{-1.0}^{+1.4}$ & $1.9_{-0.2}^{+0.2}$ & $0.8_{-0.2}^{+0.2}$ & 1.5 & $4.39 / 6$ \\
\hline 17547 & $\mathrm{MCD}$ & 4.0 fixed & $0.125_{-0.011}^{+0.013}$ & $1.6_{-0.6}^{+0.8}$ & $2.0_{-0.3}^{+0.3}$ & $0.60_{-0.17}^{+0.20}$ & 0.82 & $2.10 / 7$ \\
\hline 12437 & blackbody & 4.0 fixed & $0.089_{-0.007}^{+0.008}$ & $4.2_{-1.2}^{+1.6}$ & $1.9_{-0.2}^{+0.2}$ & $0.73_{-0.18}^{+0.20}$ & 1.4 & $4.11 / 6$ \\
\hline 17547 & blackbody & 4.0 fixed & $0.105_{-0.009}^{+0.010}$ & $2.2_{-0.7}^{+0.9}$ & $1.9_{-0.3}^{+0.3}$ & $0.48_{-0.14}^{+0.17}$ & 0.76 & $3.10 / 7$ \\
\hline
\end{tabular}

Note. Parameters mean the same as in Table 3.

Assuming a hydrogen mass fraction of zero (see the supersoft ULX in M101 whose companion is likely a hydrogen depleted Wolf-Rayet star; Liu et al. 2013) and half of accretion mass going to a wind, a $30 M_{\odot}$ black hole accreting at 300-450 times of the Eddington rate matches the data. With a hydrogen mass fraction of 0.73 (solar abundance), the best match suggests a black hole mass of $\sim 50 M_{\odot}$ at similar accretion rates (not shown in the plot). If a smaller fraction of mass is transferred to the wind, a higher accretion rate is required. These estimates may be quantitatively inaccurate, because of the simplification of the model and a deflected $R-T$ slope. Qualitatively, a picture involving a stellar mass black hole with supercritical accretion matches the observations.

The source light curve for intrinsic luminosity in the energy range of $0.3-2 \mathrm{keV}$ is shown in Figure 6. We note that for each individual Swift observation, the luminosity is converted from the net count rate based on the best-fit model in the associated observation group (Table 5). The fractional Poisson error on the net count rate is adopted for Swift data and is certainly an underestimate. For XMM-Newton and Chandra data, the luminosities and errors are directly computed from their bestfit models using the cflux model in XSPEC. The source is not as variable as other soft ULXs, like the one in M101. No specific pattern or period can be found in the current light curve.

\subsection{Short-term X-Ray Variability}

The Fourier power spectrum in the energy band of $0.3-2 \mathrm{keV}$ for the 2009 XMM-Newton observation was reported in Jin et al. (2011). Here we include timing analysis of the 2014 observation. The power spectrum was generated from time intervals free of background flares and timing gaps, using PN events in the source region with FLAG $=$ \#XMMEA_EP and PATTERN $\leqslant 4$. The light curves were generated with a resolution of the CCD frame time $(\sim 73 \mathrm{~ms})$ and divided into short segments for 8192-point Fourier transforms. The final power spectrum density was obtained by coadding spectra from individual segments, normalized to root mean square (rms) with Poisson noise subtracted, and binned in logarithmic scale on frequency.

The power spectra for the 2009 observation and for the 2014 observation during the high (non-dipping) state are displayed in Figure 7 for comparison. For the dipping intervals, there are not sufficient counts for timing analysis. It is obvious that the source displayed huge short-term variability in the 2009 observation (low state) but much less in 2014 (high state). The fractional rms variability is $0.49 \pm 0.03$ in 2009 (low state) and $0.12 \pm 0.02$ in 2014 (high state), both in the frequency range of $1.66 \mathrm{mHz}$ to $0.01 \mathrm{~Hz}$ and in the energy range of $0.3-2 \mathrm{keV}$. The variability in the 2009 observation was energy dependent (Jin et al. 2011) with a fractional rms of $0.33 \pm 0.04$ in the $0.3-0.8 \mathrm{keV}$ band and $0.76 \pm 0.07$ in the $0.8-2 \mathrm{keV}$ band. For the high state in the 2014 observation, the variability seems to be energy independent, with a fractional rms of $0.15 \pm 0.03$ in the $0.3-0.8 \mathrm{keV}$ band and $0.11 \pm 0.03$ in the $0.8-2 \mathrm{keV}$ band. At energies above $2 \mathrm{keV}$, no significant power or meaningful upper limit can be obtained due to the low statistics for both observations.

\subsection{Spectral Modeling to the Optical Data}

The measured HST spectrum composed of the WFC3 and SBC data is shown in Figure 8. A combination of two blackbody components subject to reddening (Cardelli et al. 1989) can fit the spectrum, resulting in $\chi^{2}$ of 91.7 with 7 degrees of freedom. We note that most of the residuals come from the two IR data points. Excluding the IR data gives $\chi^{2}$ of 6.7 with 5 degrees of freedom for the same model. Of course the fit can be improved by adding more spectral components, but that is hard to justify based on two data points. As we mentioned above, the IR flux may have extra uncertainties due to systematic errors in photometry. If we assume a systematic error of $10 \%$ in flux, the fitting is acceptable with $\chi^{2}$ of 13.9 and 7 degrees of freedom. We adopt this as the formal results and the best-fit model parameters are listed in Table 6. For comparison, a reddened power-law model gives a poor fit with a $\chi^{2}$ of 547 with 10 degrees of freedom.

Even at extremely high accretion rates, the outer part of the accretion disk is still thought to keep the form predicted by the standard accretion disk model (Shakura \& Sunyaev 1973), which predicts a power-law spectrum, $F_{\nu} \propto \nu^{1 / 3}$, in the optical band and cuts off at $\nu_{\text {out }}$ corresponding to the temperature of the blackbody emission at the outermost radius $R_{\text {out }}$. If irradiation from the inner disk onto the outer disk is taken into account (e.g., Gierliński et al. 2009; Sutton et al. 2014), it changes the radial temperature profile and also predicts a power-law-like spectrum at frequencies above $\nu_{\text {out }}$, except that the spectral energy distribution displays a bump before rolling down at $\nu_{\text {out }}$. To test if the observed blackbody-like optical spectrum is a result of disk irradiation, we fit the optical data below $10000 \AA$ with a self irradiated standard disk model (Madhusudhan et al. 2008) with three parameters: the X-ray (irradiating) luminosity, and the inner and outer disk radius. We will show later in the discussion section that the MCD model used to fit the soft thermal spectrum is invalid. If the inner accretion disk is covered by optically thick outflows, the disk inner radius cannot be directly measured from the spectrum. We thus fix the inner radius at $10^{7} \mathrm{~cm}$ typically for a $10 M_{\odot}$ stellar mass black hole. The best-fit results are shown in Figure 9, with an 
Table 5

Spectral Modeling to the Swift/XRT Spectra

\begin{tabular}{|c|c|c|c|c|c|c|c|c|c|}
\hline Group & $\begin{array}{l}\text { ObsID } \\
(000334690+)\end{array}$ & Model & $\begin{array}{c}N_{\mathrm{H}, \mathrm{ext}} \\
\left(10^{21} \mathrm{~cm}^{-2}\right)\end{array}$ & $\begin{array}{c}k T_{\mathrm{th}} \\
(\mathrm{keV})\end{array}$ & $\underset{\left(10^{4} \mathrm{~km}\right)}{R_{\mathrm{th}}}$ & $\begin{array}{c}f_{0.3-10 \mathrm{keV}} \\
\left(10^{-13} \mathrm{cgs}\right)\end{array}$ & $\begin{array}{l}L_{0.3-10 \mathrm{keV}} \\
\left(10^{40} \mathrm{cgs}\right)\end{array}$ & $\begin{array}{c}L_{\mathrm{th}, \mathrm{bol}} \\
\left(10^{40} \mathrm{cgs}\right)\end{array}$ & $\chi^{2} /$ dof \\
\hline 1 & $01 / 02 / 06$ & $\mathrm{MCD}$ & 4.0 fixed & $0.160_{-0.015}^{+0.017}$ & $0.9_{-0.3}^{+0.4}$ & $4.0_{-0.6}^{+0.6}$ & $0.71_{-0.17}^{+0.19}$ & 0.73 & $1.50 / 6$ \\
\hline 2 & $03 / 04 / 10 / 26$ & MCD & 4.0 fixed & $0.139_{-0.013}^{+0.014}$ & $1.4_{-0.5}^{+0.6}$ & $3.4_{-0.5}^{+0.5}$ & $0.8_{-0.2}^{+0.2}$ & 0.96 & $4.36 / 6$ \\
\hline 3 & $05 / 15 / 16 / 20 / 22$ & MCD & 4.0 fixed & $0.17_{-0.02}^{+0.02}$ & $0.6_{-0.2}^{+0.3}$ & $2.5_{-0.4}^{+0.4}$ & $0.39_{-0.11}^{+0.13}$ & 0.38 & $5.87 / 5$ \\
\hline 4 & $07 / 08 / 18 / 21 / 24 / 25 / 28$ & MCD & 4.0 fixed & $0.110_{-0.009}^{+0.010}$ & $2.8_{-0.8}^{+1.1}$ & $2.3_{-0.4}^{+0.4}$ & $0.9_{-0.2}^{+0.2}$ & 1.50 & $3.58 / 5$ \\
\hline 5 & $09 / 11 / 12 / 13 / 14 / 17 / 19 / 23 / 27$ & $\mathrm{MCD}$ & 4.0 fixed & $0.142_{-0.012}^{+0.014}$ & $0.9_{-0.3}^{+0.3}$ & $1.5_{-0.2}^{+0.2}$ & $0.34_{-0.08}^{+0.09}$ & 0.40 & $4.05 / 6$ \\
\hline 3 & $05 / 15 / 16 / 20 / 22$ & blackbody & 4.0 fixed & $0.138_{-0.014}^{+0.017}$ & $0.9_{-0.3}^{+0.4}$ & $2.5_{-0.4}^{+0.4}$ & $0.32_{-0.09}^{+0.11}$ & 0.41 & $7.83 / 5$ \\
\hline 4 & $07 / 08 / 18 / 21 / 24 / 25 / 28$ & blackbody & 4.0 fixed & $0.094_{-0.007}^{+0.007}$ & $3.8_{-1.0}^{+1.3}$ & $2.3_{-0.4}^{+0.4}$ & $0.78_{-0.19}^{+0.21}$ & 1.40 & $3.01 / 5$ \\
\hline 5 & $09 / 11 / 12 / 13 / 14 / 17 / 19 / 23 / 27$ & blackbody & 4.0 fixed & $0.117_{-0.009}^{+0.010}$ & $1.3_{-0.4}^{+0.5}$ & $1.5_{-0.2}^{+0.2}$ & $0.29_{-0.07}^{+0.08}$ & 0.42 & $3.46 / 6$ \\
\hline
\end{tabular}

Note. Parameters mean the same as in Table 3.
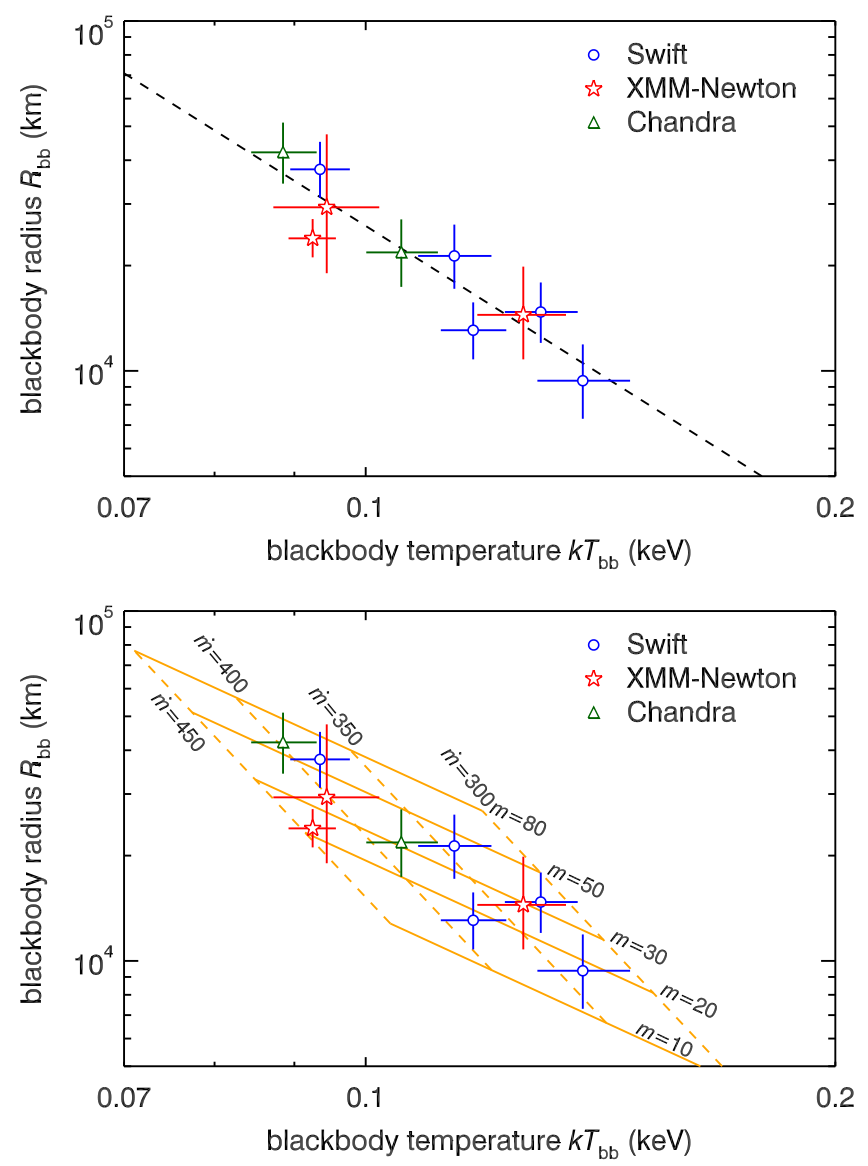

Figure 5. Blackbody radius vs. temperature for NGC 247 ULX. Top: data and linear regression (dashed line) with a slope of $-2.8 \pm 0.3$. Bottom: data and predicted radius-temperature relations from an outflow model (Soria \& Kong 2016) assuming depleted hydrogen and half of the accretion mass channeled to wind. $m$ is the black hole mass in units of $M_{\odot}$, and $\dot{m}$ is the mass accretion rate in units of the Eddington rate. Solid lines represent $R-T$ relations given a black hole mass of $10,20,30,50$, or $80 M_{\odot}$, and each dashed line connects black holes of different masses at the same accretion rate, at 300, 350, 400 , or 450 times the Eddington rate.

irradiating luminosity of $\sim 10^{41} \mathrm{erg} \mathrm{s}^{-1}$ and an outer radius of $\sim 10^{12} \mathrm{~cm}$.

If we consider the WFC3 data only, a power-law model $\left(F_{\lambda} \propto \lambda^{-2.9 \pm 0.1}\right.$ with $\left.E(B-V)=0.18 \pm 0.02\right)$ provides a

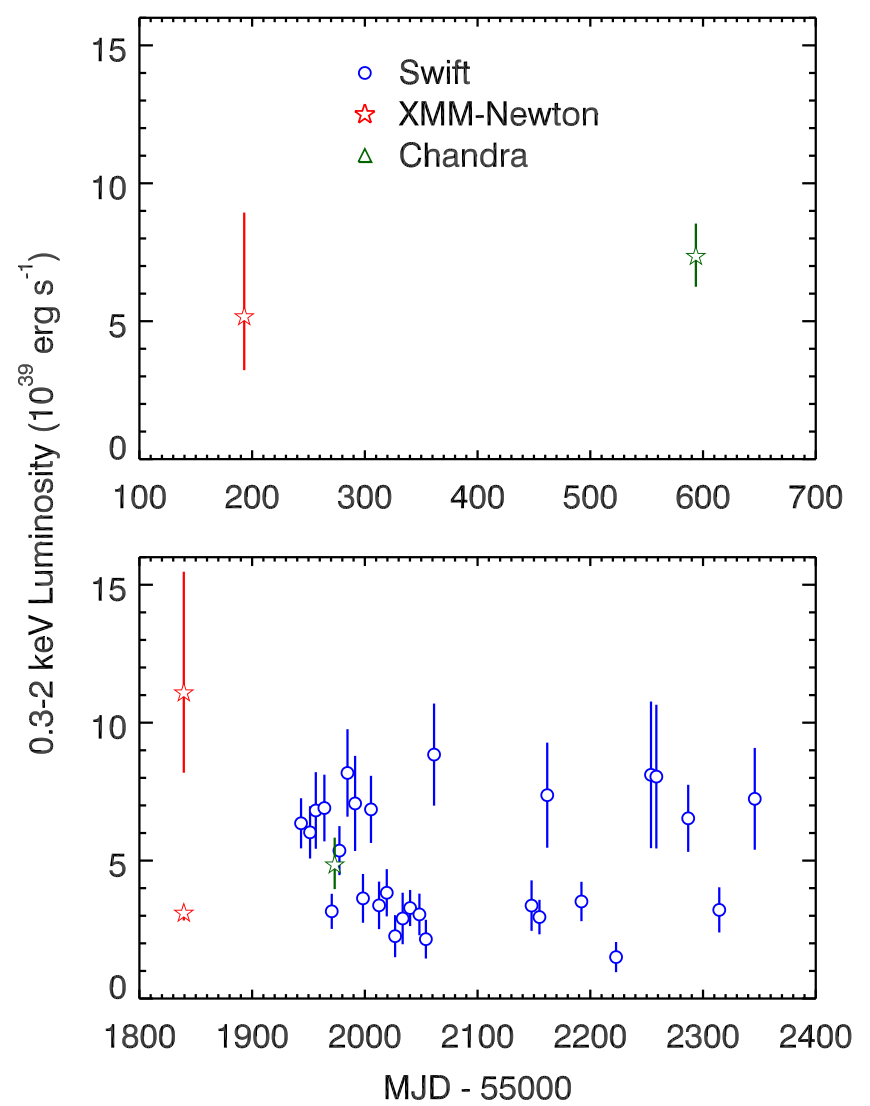

Figure 6. Long-term X-ray light curve of NGC 247 ULX in the energy range of $0.3-2 \mathrm{keV}$.

better fit than a blackbody, with a $\chi^{2}$ of 8.2 versus 97.0 with 4 degrees of freedom. However, the reddened power-law model predicts a UV flux higher than the observed flux in the ACS SBC band by a factor of 1.7-3.5. Inclusion of UV data below $2000 \AA$ is key in determining the optical emission mechanism.

\section{DISCUSSION}

These multiwavelength and multi-epoch observations provide insight into the nature of the soft ULX in NGC 247. The physical interpretation of the data and results are briefly discussed in the following. 


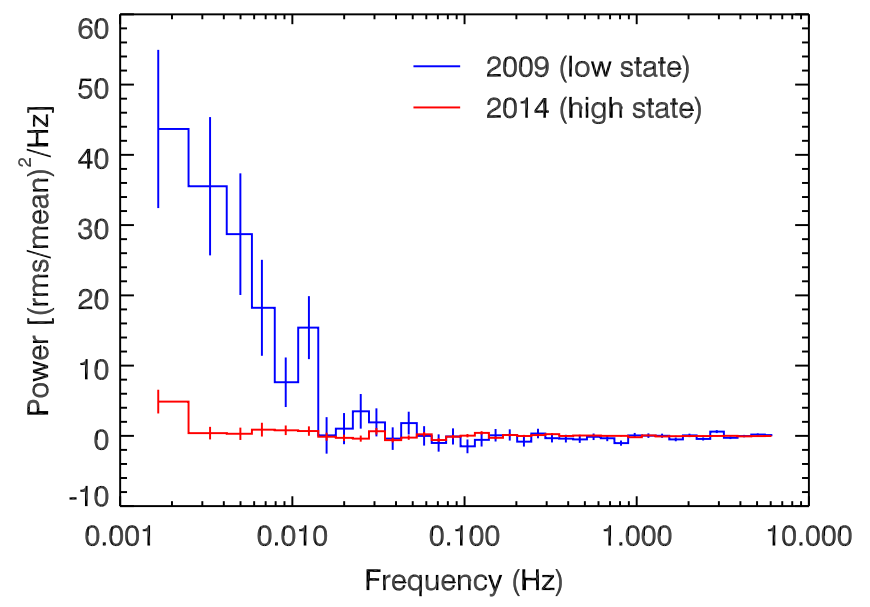

Figure 7. X-ray power spectrum density in the $0.3-2 \mathrm{keV}$ band from the 2009 $X M M$-Newton observation (blue), where the flux is relatively low, and from the 2014 observation during the non-dipping state (red), where the flux is relatively high.

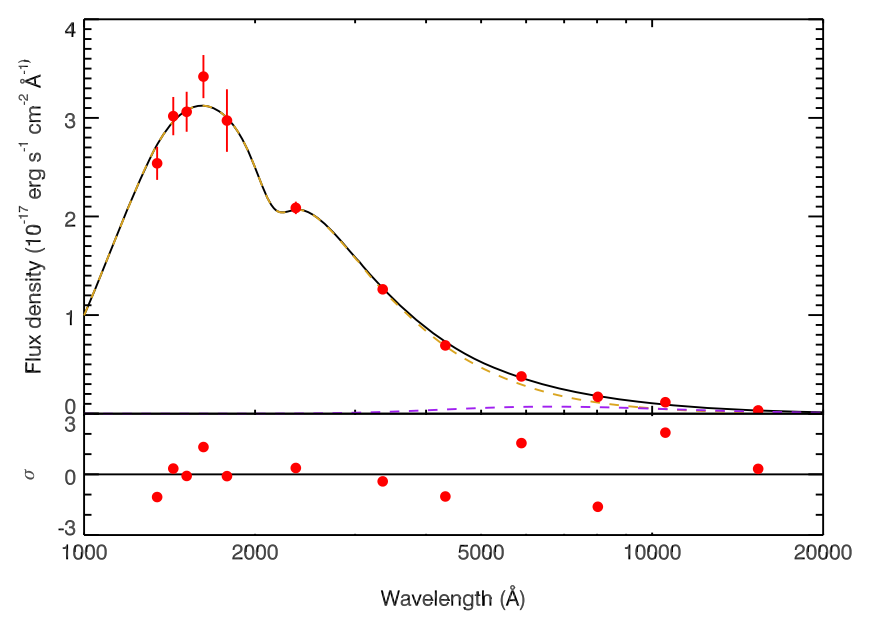

Figure 8. Measured HST spectrum for the ULX using WFC3 photometry and ACS/SBC grism data. The model (solid black) consists of two blackbody components (dashed yellow and purple), see Table 6.

Table 6

Model Parameters Fitted to the HST Spectra

\begin{tabular}{ll}
\hline \hline Parameter & Value \\
\hline Extinction $E(B-V)$ & $0.085 \pm 0.017$ \\
Blackbody temperature $(\mathrm{K})$ & $19000 \pm 400$ \\
Blackbody area $\left(\mathrm{cm}^{2}\right)$ & $(2.5 \pm 1.7) \times 10^{25}$ \\
Blackbody temperature $(\mathrm{K})$ & $4600 \pm 400$ \\
Blackbody area $\left(\mathrm{cm}^{2}\right)$ & $(4.5 \pm 1.6) \times 10^{26}$ \\
$\chi^{2} /$ dof & $13.9 / 7$ \\
\hline
\end{tabular}

Note. Assuming a $10 \%$ uncertainty on the WFC3/IR flux. An assumption of $20 \%$ will change the $\chi^{2}$ to 9.4 but do not affect the derived error bounds, which are quoted at a $1 \sigma$ confidence level.

\subsection{Transition between the Supersoft and Soft Ultraluminous Regimes}

In contrast to previous observations, soft blackbody emission does not dominate the 2014 high-state spectrumthe blackbody and power-law components exhibit almost the same luminosity in the $0.3-10 \mathrm{keV}$ band. The power-law

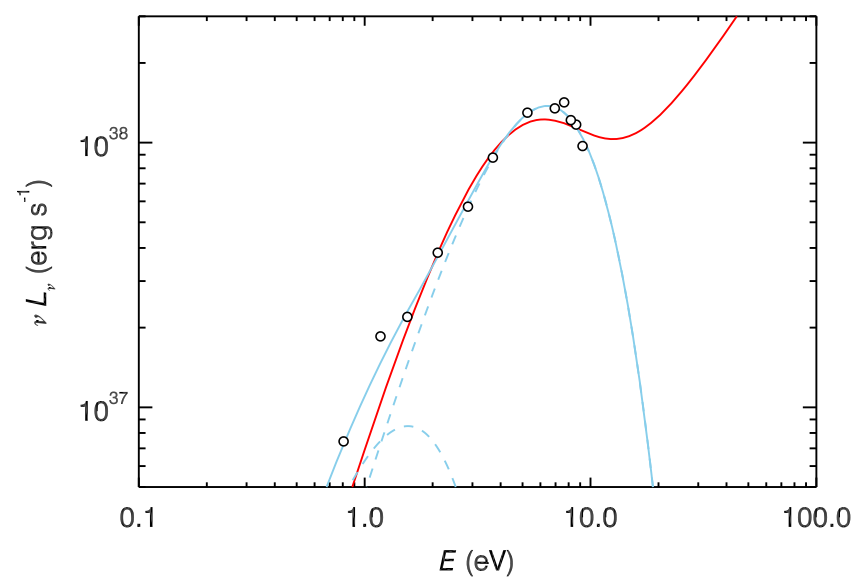

Figure 9. Fitting the optical data with a self-irradiated standard accretion disk model (red). The circles are unreddened optical data and blue lines are the twocomponent blackbody model fitted to the data (Figure 8).

component is much stronger than it was in the spectrum of other soft ULXs and NGC 247 ULX in the low state. Also, the strong fast variability previously seen in the X-ray low state is also substantially depressed in the X-ray high state. Both the spectral and temporal properties in the 2014 high state are consistent with those defined for the soft ultraluminous (SUL) regime (Sutton et al. 2013), except that the power-law component is relatively weaker and steeper and the soft blackbody component is relatively more variable on short timescales. The X-ray high state of NGC 247 ULX may be an extreme case of the SUL regime (Sutton et al. 2013).

The X-ray low state of NGC 247 ULX, both in spectral and timing properties, defines a distinct accretion regime. We call it the supersoft ultraluminous (SSUL) regime, with a spectrum characterized by a dominant soft blackbody component typically of $0.1 \mathrm{keV}$ in temperature and $10^{4}-10^{5} \mathrm{~km}$ in radius. In the SSUL regime, prominent short-term variability may be associated with the soft X-rays, also detected in M101 ULX (Mukai et al. 2003, 2005; Soria \& Kong 2016; Urquhart \& Soria 2016). For both sources in the SSUL regime, the variability is scaled with energies with a larger fractional rms seen in higher energies toward $2 \mathrm{keV}$ (the timing behavior is unknown for photons above $2 \mathrm{keV}$ ), which is similar to the behavior of NGC 5408 X-1 and NGC 6946 X-1 (HernándezGarcía et al. 2015). The origin of the fast variability seen in the SSUL regime is unclear. Due to the large size $\left(10^{4}-10^{5} \mathrm{~km}\right)$ of the blackbody emitting region and the high amplitude $(\sim 50 \%)$ of the time variation associated with it, it seems difficult to explain it with the same scenario applied for the SUL regime, that the turbulence in the wind causes the variability by scattering hard X-rays out of the line of sight (Takeuchi et al. 2013). We speculate that the SSUL regime has an accretion rate that is even higher than in the SUL/HUL regimes. SSUL sources may transition to SUL when the accretion rate decreases. Urquhart \& Soria (2016) predicted that such a transition may occur when the blackbody temperature is above $\sim 150 \mathrm{eV}$ and that is indeed observed in this source.

An alternative interpretation is that the SSUL regime is simply due to a geometric effect, being a standard broadband ULX viewed at a high inclination angle (also see discussions in 
Urquhart \& Soria 2016). The temperature of the photosphere is uneven, perhaps hotter near the disk plane and cooler outside. An occulter with a size comparable to the photosphere, such as a warped disk or a circumbinary disk far outside, may obscure the hotter region of the photosphere, leading to a lower temperature and flux. The motion of the occulter may be the source of the strong variability.

There is no solid evidence for or against either speculation. The link between the absorption edge and the strong variability may be explained by both as being due to increased wind caused by increased accretion or related to obscuration. The power spectrum density in a $\nu^{-1}$ shape consistent with that produced by viscous instability, along with the negative $R-T$ relation, is in favor of the high accretion scenario. Similar to NGC 247 ULX, NGC 5408 X-1 displays a negative rms-flux relation on long timescales of years (Caballero-García et al. 2013), except that their measurements were made in the energy range of $1-10 \mathrm{keV}$. The energy dependence of the rms variability below $2 \mathrm{keV}$ is also similar for the two sources. These similarities seem to be in support of the viewing angle interpretation. More observations and studies of a large sample is needed to further constrain the nature of these supersoft ULXs.

On the contrary, variable ULXs, like NGC 5408 X-1 and NGC 6946 X-1, show a positive rms-flux relation on short timescales, over which the timing and spectral properties are stationary (Heil \& Vaughan 2010; Hernández-García et al. 2015); this relation is found to be ubiquitous in bright accreting objects. A direct comparison can be made between this ULX and NGC 5408 X-1 if the short-term rms-flux relation can be measured in the future.

\subsection{Invalidity of the Disk Interpretation}

The thermal state of black hole binaries is characterized by a spectrum consisting of a dominant thermal MCD component plus a minor power law or Comptonization component (Remillard \& McClintock 2006). It has been suggested that the thermal spectrum seen in these soft ULXs could be due to emission from a standard accretion disk surrounding an intermediate-mass black hole. For most other soft ULXs, this has already been ruled out because the pattern of their spectral evolution (e.g., decreasing radius with increasing temperature; Soria \& Kong 2016; Urquhart \& Soria 2016) is inconsistent with that seen in the thermal state (constant inner radius with varying temperature). For NGC 247 ULX, previous studies could not rule this out due to insufficient data. Here, multi-epoch observations with XMM-Newton, Chandra, and Swift (Figure 5) identify a similar spectral evolution pattern, $R_{\mathrm{bb}} \propto T_{\mathrm{bb}}^{-2.8 \pm 0.3}$, which is steeper than that found for the ULX in M101 (slope 2; Soria \& Kong 2016) and for the whole population of soft ULXs (slope = $-2.2 \pm 0.5$; Urquhart \& Soria 2016), but is consistent within errors. In the spectral fitting, we had to fix the absorption column density for the Chandra and Swift data; otherwise, the error bound for the blackbody radius cannot be well constrained. Such a practice seems to be justified based on the XMM-Newton data, but the slope would change if the true absorption varies due to the degeneracy between the blackbody component and absorption.

\subsection{Connection between Soft ULXs and Soft Excesses in Broadband ULXS}

Besides the soft ULXs, similar anti-correlations have been found in broadband ULXs for their soft emission components (i.e., the soft excess) if it is modeled using an MCD model (similar results can be obtained if a blackbody model is used). For NGC 1313 X-2, Feng \& Kaaret (2007b) found a relation $L_{\text {disk }} \propto T_{\text {in }}^{-7 \pm 3}$ corresponding to $R_{\text {in }} \propto T_{\text {in }}^{-5 \pm 1}$. Feng \& Kaaret (2009) observed that the disk inner radius of NGC 5204 X-1 seems to shrink at higher disk inner temperatures, but the uncertainties did not allow a firm conclusion. Kajava \& Poutanen (2009) found that the soft component in broadband ULXs follow a relation $L_{\text {disk }} \propto T_{\text {in }}^{-3.5}$ or $R_{\mathrm{in}} \propto T_{\mathrm{in}}^{-4}$. Apparently, the $R-T$ relation for these soft excesses is much steeper than for the soft ULXs. However, the situation for broadband ULXs may be complicated, as a positive correlation consistent with a $L \propto T^{4}$ relation was reported for the soft component in some luminous ULXs, including NGC 5204 X-1 (Miller et al. 2013). The complexity in broadband ULXs could be due to the importance of the hard emission; how it is modeled substantially affects the parameters derived for the soft component. The treatment of absorption is also crucial to the characterization of the soft component.

Given a negative $R-T$ relation, the most plausible explanation is that these emission components may arise from the photosphere of massive outflows associated with supercritical accretion (Poutanen et al. 2007). If the $L \propto T^{4}$ relation is true, it is consistent with a scenario in which the emission comes from cool disks around more massive black holes. One of the major differences between the SSUL regime and the spectral/ timing regimes of broadband ULXs is that most soft excesses seen in broadband ULXs are less variable on short or long timescales than the soft blackbody component seen in the SSUL regime. A possible explanation may be that the blackbody-like emission from these two classes of sources may both be associated with outflows due to supercritical accretion, but they happen at different accretion rates (supersoft ULXs may have a higher accretion rate). Alternatively, as mentioned above, the two emission components may be identical under the scenario of the geometric interpretation.

\subsection{Signatures of Outflows}

In supercritical accretion models, massive outflows are a key feature predicted by theories and numerical simulations (King \& Pounds 2003; Poutanen et al. 2007; Dotan \& Shaviv 2011; Ohsuga \& Mineshige 2011; Kawashima et al. 2012; Jiang et al. 2014; King \& Muldrew 2016). Observationally, the detection of soft X-ray spectral residuals (Middleton et al. 2014, 2015b) and emission/absorption lines (Pinto et al. 2016) in ULXs have been argued as a signature of outflows associated with supercritical accretion. An absorption edge near $1 \mathrm{keV}$ is detected in the 2009 XMM-Newton observation of NGC 247 ULX, and is also detected from several other soft ULXs (Antennae, M51, M101, and NGC 4631; see Table 4 in Urquhart \& Soria 2016). Such a feature may be common in these sources and is interpreted as a result of absorption by clumpy winds (Urquhart \& Soria 2016). The edge is likely due to L-edge absorption by highly ionized iron (e.g., Fe VIII).

During the high state of the 2014 XMM-Newton observation of NGC 247 ULX, the source exhibited an emission component 
that can be explained by collisionally ionized optically thin thermal plasma. Similar features are also observed in other soft ULXs (M51 and M101; see Table 4 in Urquhart \& Soria 2016). The origin of the thermal plasma is still unknown. It may be due to shocks generated by wind-wind interaction or between the disk wind and stellar wind from the companion star. The normalization of the APEC model $\left(N_{\mathrm{APEC}} \sim 10^{-4}\right.$ in XSPEC) suggests that $\int n_{\mathrm{e}} n_{\mathrm{H}} d V=1.4 \times 10^{61}$. The thermal plasma emitting region should lie outside of the photosphere of the outflow and have a lower density than the photosphere. The density of the photosphere can be estimated as $\kappa_{\mathrm{es}} \rho_{\mathrm{ph}} r_{\mathrm{ph}} \sim 1$, where $\kappa_{\mathrm{es}} \approx 0.34 \mathrm{~g} \mathrm{~cm}^{-2}$ is the electron scattering opacity. In the 2014 high state $\left(r_{\mathrm{ph}} \approx 1.5 \times 10^{9} \mathrm{~cm}\right)$, the density of at the photosphere can be inferred as $\rho_{\mathrm{ph}} \approx 2 \times 10^{-9} \mathrm{~g} \mathrm{~cm}^{-3}$. Taking this as an upper limit, the thermal plasma emitting region has a radius of at least $10^{10} \mathrm{~cm}$. The energy of the line at $0.58 \mathrm{keV}$ is consistent with emission from highly ionized oxygen (O VII at $0.574 \mathrm{keV})$, whose cooling rate peaks at a temperature $(0.17 \mathrm{keV})$ close to the detected blackbody temperature $(0.13 \mathrm{keV})$. The density in the line emission region $n_{\mathrm{H}}=\sqrt{L_{\text {line }} /\left(5 C R^{3}\right)}$, where $L_{\text {line }} \approx 7 \times 10^{47}$ photons s $^{-1}$ is the Gaussian line luminosity and $C=2.16 \times 10^{-15}$ photons $\mathrm{cm}^{3} \mathrm{~s}^{-1}$ is the cooling rate at the blackbody temperature. Following the same assumption above that the density is lower than that at the photosphere, the line emission radius is at least $3 \times 10^{10} \mathrm{~cm}$.

The anti-correlation between the blackbody temperature and radius is predicted by outflow models. Adopting the wind model from Soria \& Kong (2016), we find that a stellar mass black hole with a mass accretion rate $\sim 10^{2}$ times the Eddington rate ( $\equiv L_{\text {Edd }} / 0.1 c^{2}$ ) seems to be a plausible solution. The inferred mass of the black hole is consistent with that measured for IC 10 X-1 (Silverman \& Filippenko 2008) and the binary black holes associated with the gravitational wave event GW150914 (Abbott et al. 2016), and can be explained as a result of core-collapse of stars of low metallicity. The accretion rate is extremely high but comparable to or lower than the estimated accretion rate for the Galactic microquasar SS 433 (Shklovskii 1981).

\subsection{Nature of the Flux Dipping}

During the 2014 XMM-Newton observation, the source displayed dips in its light curve (Figure 1). The transition between the high-flux state and the low state occurs on a timescale of $\sim 200 \mathrm{~s}$. X-ray flux dips have been observed in other ULXs, e.g., in broadband ULXs in NGC 55 (Stobbart et al. 2004), NGC 5408 (Grisé et al. 2013), and M94 (Lin et al. 2013), and also in soft ULXs in M51 and M81 (Urquhart $\&$ Soria 2016). Their nature is not determined. Here the highquality XMM-Newton data allows us to see the hardness change during the dips. It is clearly seen in Figure 1 that the blackbody temperature becomes low when entering the dip and then goes high when leaving the dip, in a manner smoother than the change of flux. The X-ray spectrum and flux in the dips are similar to those in the 2009 XMM-Newton spectrum. Both the spectral fitting and the change of hardness ratio clearly argue against the interpretation that the dips are purely due to absorption or partial absorption, further supported by the change of the blackbody temperature and emitting size (cooler and larger in the dips). Therefore, the dips are likely caused by the change of the properties of the photosphere itself. Again, a large and variable equatorial occulter could be another plausible explanation for the dips.

We estimate the physical timescales associated with dipping as follows. The wind launching radius or the spherization radius is $r_{\mathrm{sp}} \approx \dot{m} r_{\mathrm{in}}$. Assuming $\dot{m} \approx 10^{2}$ and a disk inner radius $r_{\mathrm{in}} \approx 10^{7} \mathrm{~cm}$ for a stellar mass black hole, the spherization radius is on the order of $10^{9} \mathrm{~cm}$, close to the photosphere radius of the wind, $r_{\mathrm{ph}} \approx(1.5-3) \times 10^{9} \mathrm{~cm}$. The photosphere density can be estimated assuming $\kappa_{\mathrm{es}} \rho_{\mathrm{ph}} r_{\mathrm{ph}} \sim 1$. The density increases as $r^{-2}$ and the temperature increases as $r^{-3 / 4}$ to $r^{-1 / 2}$ toward the base of the wind. If we assume a thin disk $(H / R \sim 0.1)$, then it has a thickness of $10^{8} \mathrm{~cm}$, selfconsistent in the framework of Jiang et al. (2014). From the base of the wind to the center of the disk, the ratio of the radiation pressure to the gas pressure drops by a factor of $10^{3}$ (Jiang et al. 2014). We also assume that the central disk temperature is on the order of $1 \mathrm{keV}$. This gives a viscous timescale of roughly $5 \times 10^{3} \mathrm{~s}$ and a thermal timescale of roughly $10^{2} \mathrm{~s}$ at $r \approx 10^{9} \mathrm{~cm}$. As the viscous timescale $\tau_{\text {visc }} \approx(1 / \alpha)(H / R)^{2}\left(H / c_{\mathrm{s}}\right)$ is strongly dependent on the disk thickness, for a thick disk $(H / R \sim 1)$ the viscous timescale and the thermal timescale could be on the same order of magnitude and both close to $10^{2}-10^{3} \mathrm{~s}$. However, even an order-ofmagnitude estimate would be difficult at such a high accretion rate, too little is understood about the disk structure. We note that Jiang et al. (2014) assumed an accretion rate of 22 times the Eddington rate and the simulation only reached a radius of $50 r_{\mathrm{s}} \sim 10^{8} \mathrm{~cm}$.

Therefore, it is possible that the dipping is due to the change of accretion rate on the viscous timescale or is related to some instability due to thermal effects on the wind or disk. If the accretion rate is indeed several hundred times the Eddington rate, a thick disk seems more plausible, but should be tested with future simulations.

\subsection{Optical Emission and the Companion}

For most broadband ULXs, their optical spectrum is argued to be dominated by emission from the accretion disk instead of the companion star (Tao et al. 2012b). In the optical band, they display a power-law-like continuum that can be interpreted as emission from an irradiated accretion disk (e.g., Grisé et al. 2012; Cseh et al. 2013). For NGC 247 ULX, the optical spectrum without UV data is also consistent with a power-law form with an IR excess. The SBC data rule out the power-law model, provide crucial constraints on the emission mechanism. The majority of optical emission is unlikely due to disk irradiation for NGC 247 ULX. The fitting with an irradiation model is unsuccessful especially in the UV band (Figure 9). The derived irradiating luminosity is one order of magnitude higher than the observed X-ray luminosity or the bolometric blackbody luminosity. The blackbody spectrum implies that we may see the companion star, which has a temperature of $19,000 \mathrm{~K}$ and a radius of $20 R_{\odot}$, consistent with those for a late$\mathrm{O}$ - or an early-B-type supergiant. Detection of the companion star means that this ULX could be a good target to search for stellar lines from the companion for a dynamical mass measurement. The presence of a second, cooler ( 4600 K) blackbody component is plausible but the evidence just based on two data points in the IR band is weak. We therefore do not discuss its nature. 


\section{CONCLUSION}

The most important observational results from multiwavelength and multi-epoch observations of NGC 247 ULX include

1. a transition from the SSUL regime to the SUL regimethis is the first time that a power-law emission with flux similar to the soft thermal emission has been detected in a supersoft ULX (for M101 ULX, the power-law component occupies only $1 / 10-1 / 5$ of the total luminosity in 0.3-10 keV; Urquhart \& Soria 2016);

2. the soft X-ray emission is highly variable on short timescales in the SSUL regime but much less variable in the SUL regime;

3. an anti-correlation between the blackbody radius and temperature, $R_{\mathrm{bb}} \propto T_{\mathrm{bb}}^{-2.8 \pm 0.3}$

4. flux dips with a transition timescale of $\sim 200 \mathrm{~s}$ that cannot be explained by an increase of absorption column density; and

5. a blackbody optical spectrum with a temperature of $19,000 \mathrm{~K}$ and a radius of $20 R_{\odot}$, subject to an extinction of $E(B-V)=0.122$.

We interpret the observations as follows.

1. Supersoft and soft ULXs may be ULXs in a special accretion regime, possibly with higher accretion rates than broadband ULXs. A standard disk explanation for the soft X-ray emission from supersoft and soft ULXs can be ruled out. An outflow model is favored, with soft $\mathrm{X}$-rays originating from the photosphere of the outflow and hard X-rays being leaked emission from the inner disk via the central funnel or advected through the wind. The presence of an absorption edge near $1 \mathrm{keV}$ or an optically thin thermal plasma emission component could be signatures of outflow; Based on the Soria \& Kong (2016) model, the NGC 247 ULX is likely a massive stellar mass black hole accreting at $\sim 10^{2}$ times of the Eddington rate. Alternatively, a scenario that supersoft ULXs being standard broadband ULXs viewed at high inclination angles cannot be ruled out; a large and variable equatorial occulter may produce the variability and dips.

2. The flux dips from NGC 247 ULX appear to be produced by changes in the photosphere of the outflow and may result from sudden increases of the accretion rate or from thermal fluctuations in the wind or disk.

3. The UV/optical spectrum if NGC 247 ULX is likely dominated by emission from the companion star, which may be an OB-type supergiant.

We thank the anonymous referee for useful comments that have helped improve the manuscript. We also thank Yanfei Jiang, Roberto Soria, Ryan Urquhart, and Li Ji for helpful discussions, and the XMM-Newton/Chandra/Swift/HST teams for successful executions of the observations. H.F. acknowledges funding support from the National Natural Science Foundation of China under grant No. 11633003, and the Tsinghua University Initiative Scientific Research Program. P. K. acknowledges partial support from STScI grant HST-GO13425

Facilities: XMM, Chandra, Swift, HST.

\section{REFERENCES}

Abbott, B. P., Abbott, R., Abbott, T. D., et al. 2016, PhRvL, 116, 061102 Abramowicz, M. A., Czerny, B., Lasota, J. P., \& Szuszkiewicz, E. 1988, ApJ, 332,646

Akritas, M. G., \& Bershady, M. A. 1996, ApJ, 470, 706

Bachetti, M., Harrison, F. A., Walton, D. J., et al. 2014, Natur, 514, 202

Brightman, M., Harrison, F., Walton, D. J., et al. 2016, ApJ, 816, 60

Caballero-García, M. D., Belloni, T. M., \& Wolter, A. 2013, MNRAS, 435,2665

Cardelli, J. A., Clayton, G. C., \& Mathis, J. S. 1989, ApJ, 345, 245

Carpano, S., Pollock, A. M. T., King, A. R., Wilms, J., \& Ehle, M. 2007, A\&A, 471, L55

Cseh, D., Grisé, F., Kaaret, P., et al. 2013, MNRAS, 435, 2896

Dewangan, G. C., Griffiths, R. E., Choudhury, M., Miyaji, T., \& Schurch, N. J. 2005, ApJ, 635, 198

Di Stefano, R., \& Kong, A. K. H. 2003, ApJ, 592, 884

Di Stefano, R., \& Kong, A. K. H. 2004, ApJ, 609, 710

Dolphin, A. E. 2000, PASP, 112, 1383

Dotan, C., \& Shaviv, N. J. 2011, MNRAS, 413, 1623

Fabbiano, G. 1989, ARA\&A, 27, 87

Fabbiano, G., King, A. R., Zezas, A., et al. 2003, ApJ, 591, 843

Farrell, S. A., Webb, N. A., Barret, D., Godet, O., \& Rodrigues, J. M. 2009, Natur, 460, 73

Feng, H., \& Kaaret, P. 2007a, ApJ, 668, 941

Feng, H., \& Kaaret, P. 2007b, ApJL, 660, L113

Feng, H., \& Kaaret, P. 2009, ApJ, 696, 1712

Feng, H., \& Kaaret, P. 2010, ApJL, 712, L169

Feng, H., \& Soria, R. 2011, NewAR, 55, 166

Gieren, W., Pietrzyński, G., Soszyński, I., et al. 2009, ApJ, 700, 1141

Gierliński, M., Done, C., \& Page, K. 2009, MNRAS, 392, 1106

Gladstone, J. C., Roberts, T. P., \& Done, C. 2009, MNRAS, 397, 1836

Grisé, F., Kaaret, P., Corbel, S., et al. 2012, ApJ, 745, 123

Grisé, F., Kaaret, P., Corbel, S., Cseh, D., \& Feng, H. 2013, MNRAS, 433, 1023

Heil, L. M., \& Vaughan, S. 2010, MNRAS, 405, L86

Hernández-García, L., Vaughan, S., Roberts, T. P., \& Middleton, M. 2015 , MNRAS, 453, 2877

Jenkins, L. P., Roberts, T. P., Warwick, R. S., Kilgard, R. E., \& Ward, M. J. 2004, MNRAS, 349, 404

Jiang, Y.-F., Stone, J. M., \& Davis, S. W. 2014, ApJ, 796, 106

Jin, J., Feng, H., Kaaret, P., \& Zhang, S.-N. 2011, ApJ, 737, 87

Kajava, J. J. E., \& Poutanen, J. 2009, MNRAS, 398, 1450

Kalberla, P. M. W., Burton, W. B., Hartmann, D., et al. 2005, A\&A, 440, 775

Kawashima, T., Ohsuga, K., Mineshige, S., et al. 2012, ApJ, 752, 18

King, A., \& Muldrew, S. I. 2016, MNRAS, 455, 1211

King, A. R., \& Pounds, K. A. 2003, MNRAS, 345, 657

Kong, A. K. H., \& Di Stefano, R. 2003, ApJL, 590, L13

Kong, A. K. H., \& Di Stefano, R. 2005, ApJL, 632, L107

Kong, A. K. H., Di Stefano, R., \& Yuan, F. 2004, ApJL, 617, L49

Lin, D., Irwin, J. A., Webb, N. A., Barret, D., \& Remillard, R. A. 2013, ApJ, 779,149

Liu, J. 2009, ApJ, 704, 1628

Liu, J., \& Di Stefano, R. 2008, ApJL, 674, L73

Liu, J.-F. 2008, ApJS, 177, 181

Liu, J.-F., Bai, Y., Wang, S., et al. 2015, Natur, 528, 108

Liu, J.-F., Bregman, J. N., Bai, Y., Justham, S., \& Crowther, P. 2013, Natur, 503,500

Madhusudhan, N., Rappaport, S., Podsiadlowski, P., \& Nelson, L. 2008, ApJ, 688,1235

Middleton, M. J., Heil, L., Pintore, F., Walton, D. J., \& Roberts, T. P. 2015a, MNRAS, 447, 3243

Middleton, M. J., Sutton, A. D., Roberts, T. P., Jackson, F. E., \& Done, C. 2012, MNRAS, 420, 2969

Middleton, M. J., Walton, D. J., Fabian, A., et al. 2015b, MNRAS, 454, 3134

Middleton, M. J., Walton, D. J., Roberts, T. P., \& Heil, L. 2014, MNRAS, 438, L51

Miller, J. M., Walton, D. J., King, A. L., et al. 2013, ApJL, 776, L36

Motch, C., Pakull, M. W., Soria, R., Grisé, F., \& Pietrzyński, G. 2014, Natur, 514, 198

Mukai, K., Pence, W. D., Snowden, S. L., \& Kuntz, K. D. 2003, ApJ, 582, 184 Mukai, K., Still, M., Corbet, R. H. D., Kuntz, K. D., \& Barnard, R. 2005, ApJ, 634, 1085

Ohsuga, K., \& Mineshige, S. 2011, ApJ, 736, 2

Pasham, D. R., Strohmayer, T. E., \& Mushotzky, R. F. 2014, Natur, 513, 74

Pence, W. D., Snowden, S. L., Mukai, K., \& Kuntz, K. D. 2001, ApJ, 561, 189 
Pinto, C., Middleton, M. J., \& Fabian, A. C. 2016, Natur, 533, 64

Poutanen, J., Lipunova, G., Fabrika, S., Butkevich, A. G., \& Abolmasov, P. 2007, MNRAS, 377, 1187

Read, A. M., \& Pietsch, W. 2001, A\&A, 373, 473

Remillard, R. A., \& McClintock, J. E. 2006, ARA\&A, 44, 49

Servillat, M., Farrell, S. A., Lin, D., et al. 2011, ApJ, 743, 6

Shakura, N. I., \& Sunyaev, R. A. 1973, A\&A, 24, 337

Shen, R.-F., Barniol Duran, R., Nakar, E., \& Piran, T. 2015, MNRAS, 447, L60

Shklovskii, I. S. 1981, SvA, 25, 315

Silverman, J. M., \& Filippenko, A. V. 2008, ApJL, 678, L17

Soria, R., \& Ghosh, K. K. 2009, ApJ, 696, 287

Soria, R., \& Kong, A. 2016, MNRAS, 456, 1837

Stobbart, A.-M., Roberts, T. P., \& Warwick, R. S. 2004, MNRAS, 351, 1063
Sutton, A. D., Done, C., \& Roberts, T. P. 2014, MNRAS, 444, 2415

Sutton, A. D., Roberts, T. P., \& Middleton, M. J. 2013, MNRAS, 435, 1758

Swartz, D. A., Ghosh, K. K., McCollough, M. L., et al. 2003, ApJS, 144,213

Swartz, D. A., Ghosh, K. K., Suleimanov, V., Tennant, A. F., \& Wu, K. 2002, ApJ, 574, 382

Takeuchi, S., Ohsuga, K., \& Mineshige, S. 2013, PASJ, 65, 88

Tao, L., Feng, H., Kaaret, P., Grisé, F., \& Jin, J. 2012a, ApJ, 758, 85

Tao, L., Kaaret, P., Feng, H., \& Grisé, F. 2012b, ApJ, 750, 110

Terashima, Y., Inoue, H., \& Wilson, A. S. 2006, ApJ, 645, 264

Terashima, Y., \& Wilson, A. S. 2004, ApJ, 601, 735

Urquhart, R., \& Soria, R. 2016, MNRAS, 456, 1859

Wilms, J., Allen, A., \& McCray, R. 2000, ApJ, 542, 914

Winter, L. M., Mushotzky, R. F., \& Reynolds, C. S. 2006, ApJ, 649, 730 